\title{
Deterministic Down-Converter and Continuous Photon-Pair Source within the Bad-Cavity Limit
}

\author{
Yue Chang, ${ }^{1}$ Alejandro González-Tudela, ${ }^{1}$ Carlos Sánchez Muñoz, ${ }^{1,2}$ Carlos Navarrete-Benlloch, ${ }^{1,3}$ and Tao Shi ${ }^{1}$ \\ ${ }^{1}$ Max-Planck Institut für Quantenoptik, Hans-Kopfermann-Strasse 1, D-85748 Garching, Germany \\ ${ }^{2}$ Física Teórica de la Materia Condensada, Universidad Autónoma de Madrid, 28049 Madrid, Spain \\ ${ }^{3}$ Institute for Theoretical Physics II, Universität Erlangen-Nürnberg, Staudtstrasse 7, 91058 Erlangen, Germany
}

(Received 2 December 2015; revised manuscript received 18 August 2016; published 10 November 2016)

\begin{abstract}
The development, characterization, and control of $N$-photon sources are instrumental for quantum technological applications. This work constitutes a step forward in this direction, where we propose a cavity quantum electrodynamics setup designed for the generation of photon pairs. We identify both the regime where our system works as a deterministic down-converter of a single input photon and as an optimal two-photon source under weak continuous driving. We use both the scattering and master equation formalisms to characterize the system, and from their connection naturally arises a physical criterion characterizing when weakly driven systems behave as continuous antibunched two-photon sources. We also show that the outgoing photons share nontrivial quantum correlations in general. We provide a specific implementation based on state-of-the-art superconducting circuits, showing how our proposal is within the reach of current technologies. As an outlook, we show the proposal can be extended to achieve deterministic conversion of a single photon into $N$ photons.
\end{abstract}

DOI: $10.1103 /$ PhysRevLett.117.203602

Single-photon sources [1] are one of the cornerstones of many quantum information protocols $[2,3]$. The success in the fabrication of these sources is built upon the simple nonlinear systems required, e.g., a two-level system [4], and a well-established characterization through the well-known second-order coherence function [5] $g^{(2)}(\tau)$ (see definition below), which yields $g^{(2)}(0)=0$ for a perfect single-photon source. The extension to $N$-photon sources lies also at the heart of many recent quantum technological applications such as the generation of NOON states [6] instrumental for quantum metrology [7], beating the diffraction limit [8], or even biological purposes $[9,10]$. There exist several methods to generate multiphoton states, e.g., probabilistic schemes using down-converted photons [11] and postselection [12-14], but at the price of an exponentially small probability. An alternative consists in using atomlike systems strongly coupled to cavities [15] or biexciton states in quantum dots systems [16-18]. The former has shown spectacular advances in the microwave regime for intracavity fields $[19,20]$, but its extension to traveling photons is so far limited to single photons [21-24]. Therefore, the efficient generation of multiphoton states is still a challenge which attracts a lot of attention, with new theoretical proposals that use Purcell enhancement on dressed atomic systems [25,26] or atomic ensembles in waveguide QED [27]. Moreover, the question on how to characterize continuous multiphoton sources in a more economical way than performing full-state tomography, is still open, with many different definitions in the literature [25,26,28-31].

In this Letter, we introduce a cavity quantum electrodynamics setup that deterministically converts a single photon into an entangled two-photon state and does so within the bad-cavity limit, that is, without requiring coherent interactions to be stronger than cavity or emitter damping. We also analyze the regime when the system is weakly driven and show that the condition for deterministic down-conversion also leads to an optimal continuous source of photon pairs. From the connection between these two regimes, we also propose a general criterion that characterizes when such weakly driven systems behave as emitters of photon pairs in well-defined pulses. Finally, we discuss possible implementations focusing on currently available circuit QED architectures and comment on the possibility to generalize our results for the generation of $N$-photon states.

Let us first consider the general scheme for a source depicted in Fig. 1(a). A nonlinear system $S$ is coupled to two (one-dimensional) baths [32]. The pump bath is used to excite the system with, e.g., a continuous driving or pulses with well-defined photon number, while the emitted light is monitored through the signal bath. Working in a picture rotating at some characteristic frequency of the system $k_{p}$ that we will choose later (using $\hbar=c=1$ ), and denoting by $\left\{p_{k}, s_{k}\right\}_{k \in \mathbb{R}}$ the annihilation operators of the baths, the Hamiltonian is given by $H=H_{S}+H_{B}+H_{S B}$, with Hamiltonians $H_{S}$ and $H_{B}=\int d k k\left(p_{k}^{\dagger} p_{k}+s_{k}^{\dagger} s_{k}\right)$ for system and bath, respectively, which interact through

$$
H_{S B}=\int d k\left(\sqrt{\frac{\gamma_{p}}{2 \pi}} p_{k}^{\dagger} a_{p}+\sqrt{\frac{\gamma_{s}}{2 \pi}} s_{k}^{\dagger} a_{s}\right)+\text { H.c. },
$$

where $a_{j}$ is the system operator that couples to the signal or pump $(j=s / p)$ bath. 


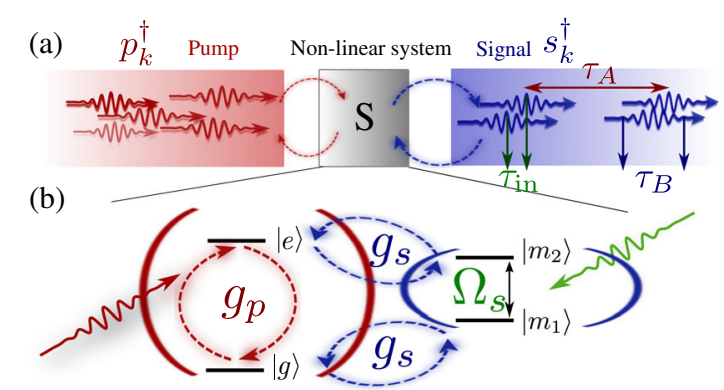

FIG. 1. Scheme for photon-pair generation. (a) The nonlinear system is driven through the pump bath (red) and the emission of photons coming out through the signal bath (blue) is analyzed. We depict the three relevant time scales that characterize the emission in our system: the intrinsic time scale of the singlephoton wave packets $\left(\tau_{B}\right)$, and the separation between the pairs $\left(\tau_{A}\right)$ and between the photons within the same pair $\left(\tau_{\text {in }}\right)$. (b) Nonlinear system that provides the interface between incoming and outgoing photons.

We consider the cavity QED model depicted in Fig. 1(b) as the system $S$, which we have designed to deterministically convert single pump photons into signal photon pairs. It consists of a four-level system with states $\{|g\rangle$, $\left.\left|m_{1}\right\rangle,\left|m_{2}\right\rangle,|e\rangle\right\}$, coupled to two photonic modes in independent cavities and a classical field. The pump mode, with annihilation operator $a_{p}$, is resonant with the $|g\rangle \leftrightarrow|e\rangle$ transition. The signal mode, on the other hand, has annihilation operator $a_{s}$ and is resonant both with $|g\rangle \leftrightarrow\left|m_{1}\right\rangle$ and $\left|m_{2}\right\rangle \leftrightarrow|e\rangle$. Finally, the classical field controls resonantly the transition $\left|m_{1}\right\rangle \leftrightarrow\left|m_{2}\right\rangle$ with a Rabi frequency $\Omega_{s}$, that will allow us to tune between different regimes of emission and, specifically, to induce deterministic down-conversion. In a picture rotating at the pump frequency, the system is then described by the Hamiltonian

$$
\begin{aligned}
H_{S}= & g_{p} a_{p}^{\dagger}|g\rangle\left\langle e\left|+\Omega_{s}\right| m_{2}\right\rangle\left\langle m_{1}\right| \\
& +g_{s} a_{s}^{\dagger}\left(\left|m_{2}\right\rangle\langle e|+| g\rangle\left\langle m_{1}\right|\right)+\text { H.c. }
\end{aligned}
$$

The scattering formalism [33] is naturally suited for analyzing processes such as the conversion of a given input state $\left|k_{1}, \ldots, k_{m}\right\rangle_{p}=p_{k_{1}}^{\dagger} \ldots p_{k_{m}}^{\dagger}|0\rangle$ with $m$ incoming pump photons with momenta $\left\{k_{1}, \ldots, k_{m}\right\}$, into an outgoing state $\left|q_{1}, \ldots, q_{n}\right\rangle_{s}=s_{q_{1}}^{\dagger} \ldots s_{q_{n}}^{\dagger}|0\rangle$ with $n$ signal photons with momenta $\left\{q_{1}, \ldots, q_{n}\right\}$. All the asymptotic information is contained in the so-called $S$ matrix, defined as $S=\lim _{t_{i} \rightarrow-\infty}^{t_{f} \rightarrow+\infty} e^{i H_{B} t_{f}} e^{-i H\left(t_{f}-t_{i}\right)} e^{-i H_{B} t_{i}}$.

Let us study first the behavior of the system when excited by a single pump photon of momentum $k_{i}$, i.e., $\left|k_{i}\right\rangle_{p}$. In order for the photon to be perfectly down-converted to a signal photon pair, the condition ${ }_{p}\left\langle k_{f}|S| k_{i}\right\rangle_{p}=0 \forall k_{f}$ must be satisfied, that is, no photons are reflected in the pump bath. We show analytically in the Supplemental Material [34] that this condition can be satisfied under resonant excitation $k_{i}=0$ for a specific control drive $\Omega_{s}=\Omega_{2 \text { ph }}$ which reads

$$
\Omega_{2 \mathrm{ph}}^{2} \approx \gamma_{s}^{2}\left[\Gamma_{s}(0)-\Gamma_{p}\right] / 4 \Gamma_{p},
$$

where we defined the Purcell-enhanced decay rates through the pump or signal cavities, $\Gamma_{p}=4 g_{p}^{2} / \gamma_{p}$ and $\Gamma_{s}\left(\Omega_{s}\right)=4 g_{s}^{2} \gamma_{s} /\left(\gamma_{s}^{2}+4 \Omega_{s}^{2}\right)$, respectively, that we obtain by adiabatically eliminating the cavity modes and the intermediate levels [34]. Interestingly, $\Omega_{2 \mathrm{ph}}$ corresponds to the driving amplitude which makes these rates equal, i.e., $\Gamma_{s}\left(\Omega_{2 \mathrm{ph}}\right)=\Gamma_{p}$. This is a similar interference effect as the one used in previous works [38-43], which we exploit here to engineer perfect down-conversion even within the bad-cavity limit $g_{j} \ll \gamma_{j}(j=p, s)$. Note that Eq. (3) requires $\Gamma_{p}<\Gamma_{s}(0)=4 g_{s}^{2} / \gamma_{s}$. Moreover, using scattering theory, we can show [34] that the reflection coefficient $\left|\int d k_{p}\left\langle k|S| k_{i}\right\rangle_{p}\right|^{2}$ has a Lorentzian shape as a function of the incident momentum $k_{i}$, with a width $\sim \Gamma_{p}+\Gamma_{s}\left(\Omega_{s}\right)$, which provides the bandwidth for efficient downconversion of single-photon pulses.

To further characterize the down-conversion process, we calculate the outgoing two-photon wave function of the signal field, defined as $\Psi_{2 \mathrm{ph}}\left(x_{1}, x_{2}\right)=\left\langle 0\left|s\left(x_{1}\right) s\left(x_{2}\right) S\right| k_{i}\right\rangle_{p}$, with $s(x)=(2 \pi)^{-1 / 2} \int d k s_{k} e^{i k x}$ annihilating signal excitations in real space. We provide its complete expression in [34], and here reproduce an approximate one in the badcavity limit and at resonance $k_{i}=0$, which reads

$$
\left|\Psi_{2 \mathrm{ph}}\left(x_{1}, x_{2}\right)\right|^{2} \propto\left|e^{-\gamma_{s} \tau}-\frac{\gamma_{s} \sin \left(\Omega_{s} \tau\right)}{2 \Omega_{s}} e^{-\Gamma_{s}\left(\Omega_{s}\right) \tau / 2}\right|^{2},
$$

where $\tau=\left|x_{1}-x_{2}\right|$. This expression shows that the wave function is indeed bunched, and therefore, the two output signal photons propagate together. Moreover, it is nonseparable, that is, the photons within the pair share entanglement. As shown in detail in [34], and following a similar route to that used in optical parametric downconversion [44], we characterize this entanglement through the Schmidt number, which allows us to perform efficient analytical calculations by assuming the input wave packet to have a Lorentzian spectral shape. We provide quantitative details in [34], and here we just want to summarize our main numerical findings: (i) the entanglement shows a linear divergence with the inverse of the spectral width of the input wave packet, and (ii) for most of the parameters, the outgoing photons show strong nontrivial quantum correlations, becoming nonentangled only for $\Omega_{s}=0$ and a specific width of the input wave packet. Note that this entanglement is different from the one of parametric sources such as those in [45], which are well described by Gaussian correlations between continuous variables.

An alternative scenario is that in which the system is continuously driven by a monochromatic laser at some 

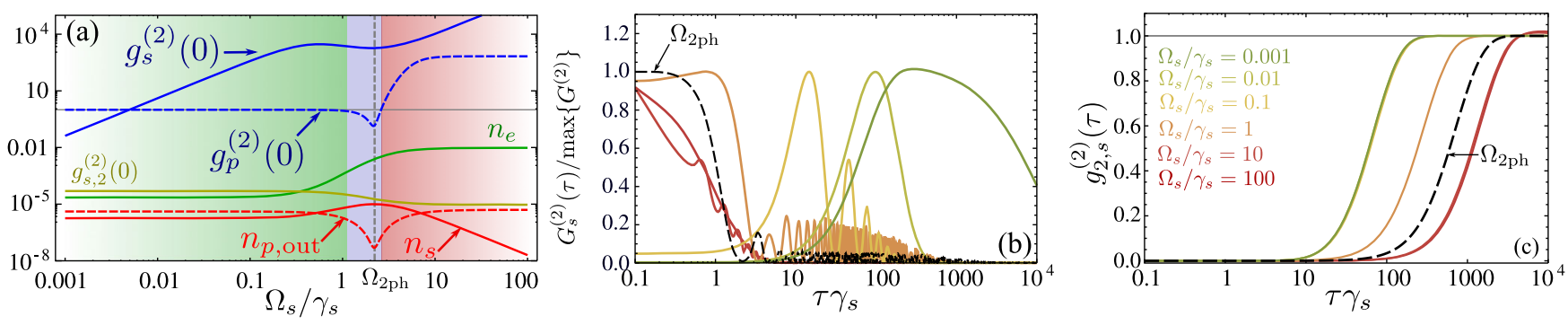

FIG. 2. (a) Main steady-state observables as a function of the control drive $\Omega_{s}$. Parameters are $\gamma_{p}=20 \gamma_{s}$, $\Omega_{p}=0.01 \gamma_{s}$, $g_{p}=g_{s}=0.1 \gamma_{s}$. (b) and (c) show, respectively, $G_{s}^{(2)}(\tau)$ (or equivalently two-photon wave function) normalized to its maximal value and $g_{s, 2}^{(2)}(\tau)$ (or equivalently four-photon wave function) as a function of $\tau$ for $\Omega_{s}$ changing logarithmically from $0.001 \gamma_{s}$ to $100 \gamma_{s}$ (in color) and for the optimal condition $\Omega_{2 \text { ph }}$ (dashed black) defined in Eq. (3). Notice that in (c) the curves from $\Omega_{s}=10^{-3}$ to 0.1 and 10 to 100 overlap. We do not show the purity of two-photon emission [25] in the plots because, due to the way the system is engineered, it is $100 \%$ for all parameters.

frequency $k_{i}$ through the pump bath (in the picture rotating with $k_{p}$ ). A standard approach in this context consists of integrating the bath degrees of freedom, which results in a master equation for the system's state $\rho[46,47]$ :

$\dot{\rho}=-i\left[H_{S}+H_{D}, \rho\right]+\sum_{j=s, p} \frac{\gamma_{j}}{2}\left(2 a_{j} \rho a_{j}^{\dagger}-a_{j}^{\dagger} a_{j} \rho-\rho a_{j}^{\dagger} a_{j}\right)$,

where $H_{D}=\Omega_{p}\left(e^{-i k_{i} t} a_{p}^{\dagger}+\right.$ H.c. $)$ is a driving term, being $\Omega_{p}$ its amplitude (taken real and positive without loss of generality). The statistics of the field emitted through the baths can be analyzed through multitime correlation functions which, using input-output theory [46,47], can be related to system correlators $G_{j}^{(n)}\left(\tau_{1}, \ldots, \tau_{n}\right)=$ $\left\langle a_{j}^{\dagger}\left(\tau_{1}\right) \ldots a_{j}^{\dagger}\left(\tau_{n}\right) a_{j}\left(\tau_{n}\right) \ldots a_{j}\left(\tau_{1}\right)\right\rangle$, where $\tau_{1}<\ldots<\tau_{n}$ with the operators defined in the Heisenberg picture and $j=s$, $p$. The master equation allows evaluating these functions via the quantum regression theorem $[46,47]$.

Even though scattering and master equation formalisms seem to apply to very different scenarios, they are very much connected $[32,48,49]$. For example, let us consider a situation in which $H$ connects pump photons with signal photon pairs, which is the case of our system. Using the various definitions provided above, we find [34] a relation between scattering amplitudes and correlation functions of such a system under weak driving. In the case of the second-order correlation function, to first nontrivial order in $\Omega_{p}$ we get [34]

$$
G_{s}^{(2)}(\tau)=\lim _{t \rightarrow \infty} G_{s}^{(2)}(t, t+\tau) \propto \Omega_{p}^{2}\left|\Psi_{2 \mathrm{ph}}\left(x_{1}, x_{2}\right)\right|^{2} .
$$

We find a similar connection between the photon-pair second-order correlation function [25] and the four-photon wave function $\Psi_{4 \mathrm{ph}}\left(x_{1}, x_{2}, x_{3}, x_{4}\right)=\langle 0| s\left(x_{1}\right) s\left(x_{2}\right) s\left(x_{3}\right) s$ $\left(x_{4}\right) S\left|k_{i}, k_{i}\right\rangle_{p}$. This reads [34]

$$
\begin{aligned}
G_{s, 2}^{(2)}(\tau) & =\lim _{t \rightarrow \infty} G_{s}^{(4)}(t, t, t+\tau, t+\tau) \\
& \propto \Omega_{p}^{4}\left|\Psi_{4 \mathrm{ph}}\left(x_{1}, x_{1}, x_{2}, x_{2}\right)\right|^{2} .
\end{aligned}
$$

Within the scattering formalism, it is clear that the system will be an ideal single photon-pair source whenever $\left|\Psi_{2 \mathrm{ph}}\left(x_{1}, x_{2}\right)\right|$ has an absolute maximum around $x_{1}=x_{2}$, while $\left|\Psi_{4 \mathrm{ph}}\left(x_{1}, x_{1}, x_{2}, x_{2}\right)\right|$ shows a wide dip at that point, meaning that the system scatters photons in well-spaced wave packets containing two signal photons. Therefore, the equivalences above naturally give rise to a physical criterion characterizing when the weakly driven system is emitting light in photon pairs: $G_{s}^{(2)}(0)>G_{s}^{(2)}(\tau)$, while keeping $G_{s, 2}^{(2)}(0)<G_{s, 2}^{(2)}(\tau)$, that is, it has to show bunching of single photons, but antibunching between photon pairs. Moreover, the antibunching time scale must be larger than the bunching one, so that the separation between the pairs is guaranteed. This connection provides, then, formal grounds to the use in weakly driven systems of the photon-pair second-order correlation function [25].

Let us now analyze the behavior of our system under resonant continuous weak driving. In Fig. 2(a) we show the dependence of the main steady-state observables on the control drive $\Omega_{s}$, as obtained from the master equation (5) or its connection with scattering theory [34], and using representative parameters within the bad-cavity limit. We represent various populations $n_{j}$ ( $j=p$ for pump, $s$ for signal, and $e$ for excited state), including the one for the output pump mode $a_{p \text {,out }}=2 \Omega_{p} / \gamma_{p}-i a_{p}$, as well as normalized correlation functions $g_{j}^{(2)}(\tau)=G_{j}^{(2)}(\tau) / n_{j}^{2}$ and $g_{s, 2}^{(2)}(\tau)=G_{s, 2}^{(2)}(\tau) /\left[G_{s}^{(2)}(0)\right]^{2}$ at $\tau=0$. We can differentiate three regimes of emission, best identified through the second-order correlation function of the pump. (i) $g_{p}^{(2)}(0)=1$, green background: This region shows a transition from $g_{s}^{(2)}(0)<1$, where the signal cavity is, therefore, emitting single photons, to $g_{s}^{(2)}(0)>1$, which 
corresponds to correlated emission from the cascade through the intermediate levels. However, when looking at the dynamics of $G_{s}^{(2)}(\tau)$ in Fig. 2(b), we can see how the maximum two-photon probability occurs at $\tau>0$, and therefore it is still not a good photon-pair source, since this indicates that the photons inside the pair are spatially separated. (ii) $g_{p}^{(2)}(0)<1$, blue background: This region shows $g_{s, 2}^{(2)}(0)<g_{s, 2}^{(2)}(\tau)$ as shown in Fig. 2(c), maximal $G_{s}^{(2)}(\tau)$ very close to $\tau=0$, and a bunching time scale much shorter than the antibunching one of $g_{s, 2}^{(2)}(\tau)$. Therefore, photons inside a pair are emitted together and the pairs are well separated, so the system behaves as a good photon-pair source according to the criterion defined above. Moreover, this region features a maximal signal population $n_{s}$ (and minimal $n_{p \text {,out }}$ ) at the optimum control drive $\Omega_{2 \mathrm{ph}}$, yielding then a maximum photon-pair emission rate given by $\gamma_{s} n_{s}$. (iii) $g_{p}^{(2)}(0)>1$, red background: With photon-pair emission but with a decrease of its rate.

In order to gain an understanding of the two-photon emission process, we analyze the relevant time scales for the emission of photon pairs, which are schematically depicted in Fig. 1(a), and we define in what follows. Once the system arrives at $|e\rangle$ it relaxes to $|g\rangle$ in a time $\tau_{A}$ (acting then as the reloading time), either emitting a pump photon or two cascaded signal photons separated by a time $\tau_{\text {in }}$. Denoting by $\tau_{B}$ the intrinsic width of the single-photon wave packets, it is then clear that $\tau_{\text {in }}<\tau_{B}<\tau_{A}$ is required for the system to act as an antibunched two-photon source. We have made a detailed analysis of these time scales [34], and here we focus on the results found within the badcavity limit $\left(g_{j} \ll \gamma_{j}\right)$ and with a strong-enough control drive $\left(\Omega_{s} \gg g_{j}^{2} / \gamma_{j}\right)$. In this regime, it is shown that $\tau_{\text {in }}$ scales as $\Omega_{s}^{-1}$, and hence the delay between photons within the same pair can be made arbitrarily small by increasing the control drive. Under such circumstances, we get $\tau_{B} \sim \gamma_{s}^{-1}$, which determines the time scale of the bunching in $G_{s}^{(2)}(\tau)$, and provides the requirement $\Omega_{s}>\gamma_{s}$. Finally, $\tau_{A}$ is determined by the decay rate from $|e\rangle$ to $|g\rangle$ through the $p$ and $s$ channels, leading to $\tau_{A}^{-1} \approx \Gamma_{p}+\Gamma_{s}\left(\Omega_{s}\right)$. The different dependence of the scaling of these time scales with $\Omega_{s}$ explains the transition between the different regimes in Fig. 2.

To further estimate the feasibility of our proposal, we now analyze the effect of having spontaneous emission from $|e\rangle$ to $|g\rangle$ through other dissipative channels. Assuming such processes to occur at the rate $\gamma^{*} \ll \gamma_{p, s}$, this time scale contributes to the reloading time as $\tau_{A}^{-1} \approx$ $\Gamma_{s}\left(\Omega_{s}\right)+\Gamma_{p}+\gamma^{*}$ [34]. It is then clear that as long as $\gamma^{*} \ll \gamma_{s}$, the condition $\tau_{A} \ll\left\{\tau_{B}, \tau_{\text {in }}\right\}$ will then still be satisfied. Such an intuitive result is confirmed numerically [34], where we see that, as expected, by increasing $\gamma^{*}$ above $\gamma_{s}$ the system shows a transition from antibunched to bunched photon pairs, as $\tau_{A}$ becomes comparable to $\tau_{B}$. (a)

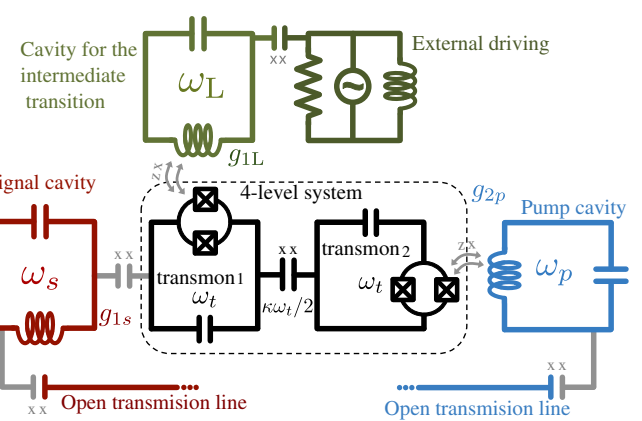

(b)

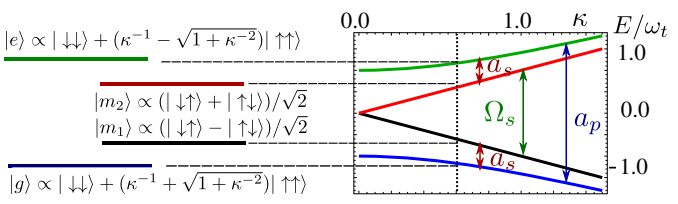

FIG. 3. (a) Circuit QED implementation: two coupled transmon qubits provide the desired four-level structure (b), while three $L C$ circuits provide the single-mode cavities playing the role of signal, pump, and the classical driving for the intermediate transition. The baths are implemented with open transmission lines.

One versatile platform to implement our ideas is circuit QED [50-52], where we can use long-lived qubits, singlemode cavities, and open transmission lines to design our proposed setup. For concreteness, a specific architecture is depicted in Fig. 3(a), although it is important to note that any other architecture containing the ingredients and couplings that we introduce would work as well, as our ideas do not rely on the details of the implementation. Two identical qubits (transmons $[53,54]$ in the figure, details can be found in [34]) with energies $\omega_{t}$ are capacitively coupled through an $x x$ interaction $\omega_{t} \kappa \sigma_{x}^{(1)} \sigma_{x}^{(2)} / 2$, whose spectrum is shown in Fig. 3(b). The desired four level structure appears between the states $\left|m_{2,1}\right\rangle \propto|\uparrow \downarrow\rangle \pm|\downarrow \uparrow\rangle$ and $|e, g\rangle \propto\left(\kappa^{-1} \mp \sqrt{1+\kappa^{-2}}\right)|\uparrow \uparrow\rangle+|\downarrow \downarrow\rangle$, with respective energies $E_{2,1}= \pm \omega_{t} \kappa$ and $E_{e, g}= \pm \omega_{t} \sqrt{1+\kappa^{2}}$. Here, $|\downarrow\rangle$ and $|\uparrow\rangle$ are the ground and excited qubit states. Two singlemode $L C$ resonators with frequencies $\omega_{p}=E_{e}-E_{g}$ and $\omega_{s}=E_{e}-E_{2}=E_{1}-E_{g}$ provide the pump and signal modes, respectively, while an additional strongly driven resonator with frequency $\omega_{L}=E_{2}-E_{1}$ is used to control the intermediate transition. The pump and classical cavities are inductively coupled each to one transmon via $x z$ interactions, $g_{2 p}\left(a_{p}+a_{p}^{\dagger}\right) \sigma_{z}^{(2)} \quad$ and $\quad g_{1 \mathrm{~L}}\left(a_{L}+a_{L}^{\dagger}\right) \sigma_{z}^{(1)}$. Finally, the signal cavity is capacitively coupled through an $x x$ interaction to one of the transmons, $g_{1 s}\left(a_{s}+a_{s}^{\dagger}\right) \sigma_{x}^{(1)}$. Working in the $\kappa \ll 2$ regime and provided $\left\{g_{1 \mathrm{~L}} \alpha, g_{2 p} \kappa, g_{1 s} / \sqrt{2}\right\} \ll 2 \omega_{t} \kappa$, these types of couplings ensure that in the eigenbasis of the two-qubit system the full system Hamiltonian takes the form of Eq. (2), with $\Omega_{s}=g_{1 \mathrm{~L}} \alpha, g_{p}=-g_{2 p} \kappa$, and $g_{s}=g_{1 s} / \sqrt{2}$ [34]— $\alpha$ being the number of excitations in the classical cavity that can be controlled via the external driving. Using $\gamma_{p, s}$ on the tens of $\mathrm{MHz}$ range, the spontaneous emission of the 
superconducting qubits gets orders of magnitude below such large cavity decay rates, while the cooperativities $C_{j}=4 g_{j}^{2} / \gamma_{j} \gamma^{*}(j=p, s)$ can be made very large since couplings $g_{2 p}$ and $g_{1 s}$ up to tens of $\mathrm{MHz}$ are routinely achieved in current experiments. With these parameters, at the optimal point $\Omega_{s}=\Omega_{2 \mathrm{ph}}$, the rate of photon-pair emission $\gamma_{s} n_{s}$ can get up to the $0.1-10 \mathrm{kHz}$ range for $\Omega_{p} / \gamma_{s} \in\left[10^{-2}, 10^{-1}\right]$.

Other platforms may fulfill our requisites in the optical domain, such as natural or artificial atoms using its "butterflylike" level structure, coupled to nanophotonic cavities. Current experiments with atoms [55] show cooperativities around 10, with decay rates up to $25 \mathrm{GHz}$, which would lead to photon-pair emission rates in the $0.1-10 \mathrm{MHz}$ range, which already exceed current parametric down-conversion technologies.

Summing up, we have designed a cavity QED setup that acts as a deterministic down-converter when excited by single photons or as a continuous entangled photon-pair source when weakly driven, and does so within the badcavity limit. From the connection between the two regimes, we also formalized a criterion characterizing photon-pair sources under weak driving, based on the dynamics of the standard correlation function $g^{(2)}(\tau)$ and the generalized one of the pairs, $g_{2}^{(2)}(\tau)$, first introduced in [25]. Our analysis of the figures of merit and scaling with different parameters has shown the feasibility of the proposal, for which we have designed a concrete implementation based on superconducting circuits. We believe that our characterization, analysis, and implementation proposal represent an important step forward in the fabrication of efficient two-photon sources. We also foresee its extension to $N$-photon states, and, as a first step, we show in [34] how to obtain deterministic conversion of single photons into $N$ photons, by using more elaborate schemes with $2 N$ levels. We will analyze such setups in depth in future works.

We thank Peter Zoller, Eugenio Roldán, and J. Kofler for useful suggestions. We also appreciate the input of the anonymous referee, who encouraged us to characterize the entanglement properties of the photon pairs. This work has been funded by the European Union integrated project SIQS. A. G.-T. acknowledges support from the IntraEuropean Marie-Curie Fellowship NanoQuIS (625955). C. S. M. is supported by the FPI programme of the Spanish MINECO through Projects No. MAT2011- 22997 and No. MAT2014-53119-C2-1-R.

All the authors contributed equally to the project.

Note added.-Recently, a preprint appeared proposing an alternative route to deterministic down-conversion [43].

[1] B. Lounis and M. Orrit, Rep. Prog. Phys. 68, 1129 (2005).

[2] J. L. O’Brien, Science 318, 1567 (2007).
[3] J. L. O'Brien and J. V. Akira Furusawa, Nat. Photonics 3, 687 (2009).

[4] C. Brunel, B. Lounis, P. Tamarat, and M. Orrit, Phys. Rev. Lett. 83, 2722 (1999).

[5] R. J. Glauber, Phys. Rev. Lett. 10, 84 (1963).

[6] I. Afek, O. Ambar, and Y. Silberberg, Science 328, 879 (2010).

[7] V. Giovannetti, S. Lloyd, and L. Maccone, Phys. Rev. Lett. 96, 010401 (2006).

[8] M. D’Angelo, M. V. Chekhova, and Y. Shih, Phys. Rev. Lett. 87, 013602 (2001).

[9] W. Denk, J. Strickler, and W. Webb, Science 248, 73 (1990).

[10] N. Horton, D. Wang, C. Kobat, F. Clark, C. Wise, and C. X. C. Schaffer, Nat. Photonics 7, 205 (2013).

[11] N. Bruno, A. Martin, T. Guerreiro, B. Sanguinetti, and R. T. Thew, Opt. Express 22, 17246 (2014).

[12] A. Ourjoumtsev, R. Tualle-Brouri, and P. Grangier, Phys. Rev. Lett. 96, 213601 (2006).

[13] A. Zavatta, V. Parigi, and M. Bellini, Phys. Rev. A 78, 033809 (2008).

[14] X.-C. Yao, T.-X. Wang, P. Xu, H. Lu, G.-S. Pan, X.-H. Bao, C.-Z. Peng, C.-Y. Lu, Y.-A. Chen, and J.-W. Pan, Nat. Photonics 6, 225 (2012).

[15] C. K. Law and J. H. Eberly, Phys. Rev. Lett. 76, 1055 (1996).

[16] G. Callsen, A. Carmele, G. Hönig, C. Kindel, J. Brunnmeier, M. R. Wagner, E. Stock, J.S. Reparaz, A. Schliwa, S. Reitzenstein, A. Knorr, A. Hoffmann, S. Kako, and Y. Arakawa, Phys. Rev. B 87, 245314 (2013).

[17] A. Dousse, J. Suffczyński, A. Beveratos, O. Krebs, A. Lemaître, I. Sagnes, J. Bloch, P. Voisin, and P. Senellart, Nature (London) 466, 217 (2010).

[18] M. Müller, S. Bounouar, K. D. Jöns, M. Glässl, and P. Michler, Nat. Photonics 8, 224 (2014).

[19] M. Hofheinz, E. Weig, M. Ansmann, R. Bialczak, E. Lucero, M. Neeley, A. O'Connell, H. Wang, J. Martinis1, and A. Cleland, Nature (London) 454, 310 (2008).

[20] M. Hofheinz, H. Wang, M. Ansmann, R. C. Bialczak, E. Lucero, M. Neeley, A. O’Connell, D. Sank, J. Wenner, J. M. Martinis et al., Nature (London) 459, 546 (2009).

[21] B. Johnson, M. Reed, A. Houck, D. Schuster, L. S. Bishop, E. Ginossar, J. Gambetta, L. DiCarlo, L. Frunzio, S. Girvin et al., Nat. Phys. 6, 663 (2010).

[22] D. Bozyigit, C. Lang, L. Steffen, J. M. Fink, C. Eichler, M. Baur, R. Bianchetti, P. J. Leek, S. Filipp, M. P. da Silva, A. Blais, and A. Wallraff, Nat. Phys. 7, 154 (2011).

[23] C. Eichler, D. Bozyigit, C. Lang, L. Steffen, J. Fink, and A. Wallraff, Phys. Rev. Lett. 106, 220503 (2011).

[24] M. Pechal, L. Huthmacher, C. Eichler, S. Zeytinoğlu, A. A. Abdumalikov, S. Berger, A. Wallraff, and S. Filipp, Phys. Rev. X 4, 041010 (2014).

[25] C. Sanchez Muñoz, E. del Valle, A. G. Tudela, K. Müller, S. Lichtmannecker, M. Kaniber, C. Tejedor, J. Finley, and F. Laussy, Nat. Photonics 8, 550 (2014).

[26] C. Sánchez-Muñoz, F. P. Laussy, C. Tejedor, and E. del Valle, New J. Phys. 17, 123021 (2015).

[27] A. González-Tudela, V. Paulisch, D. E. Chang, H. J. Kimble, and J. I. Cirac, Phys. Rev. Lett. 115, 163603 (2015).

[28] H.-G. Hong, H. Nha, J.-H. Lee, and K. An, Opt. Express 18, 7092 (2010). 
[29] M. Koch, C. Sames, M. Balbach, H. Chibani, A. Kubanek, K. Murr, T. Wilk, and G. Rempe, Phys. Rev. Lett. 107, 023601 (2011).

[30] A. Rundquist, M. Bajcsy, A. Majumdar, T. Sarmiento, K. Fischer, K. G. Lagoudakis, S. Buckley, A. Y. Piggott, and J. Vučković, Phys. Rev. A 90, 023846 (2014).

[31] A. Le Boité, G. Orso, and C. Ciuti, Phys. Rev. A 90, 063821 (2014).

[32] T. Shi, D. E. Chang, and J. I. Cirac, Phys. Rev. A 92, 053834 (2015).

[33] J. J. Sakurai and J. J. Napolitano, Modern Quantum Mechanics (Pearson Higher Ed., New York, 2014).

[34] See Supplemental Material at http://link.aps.org/ supplemental/10.1103/PhysRevLett.117.203602 for details of the connection between scattering and master equation formalisms, the relevant time scales of the system, the entanglement of the outgoing photon pairs, and the proposed circuit QED architecture, which also contains Refs. [35-37].

[35] L. Zhou, Z. R. Gong, Y.-x. Liu, C. P. Sun, and F. Nori, Phys. Rev. Lett. 101, 100501 (2008).

[36] J.-Q. Liao, H. K. Cheung, and C. K. Law, Phys. Rev. A 85, 025803 (2012).

[37] N. Killoran, M. Cramer, and M. B. Plenio, Phys. Rev. Lett. 112, 150501 (2014).

[38] K. Koshino, K. Inomata, T. Yamamoto, and Y. Nakamura, Phys. Rev. Lett. 111, 153601 (2013).

[39] T. C. H. Liew and V. Savona, Phys. Rev. Lett. 104, 183601 (2010).

[40] M. Bamba, A. Imamoğlu, I. Carusotto, and C. Ciuti, Phys. Rev. A 83, 021802 (2011).

[41] A. Majumdar, M. Bajcsy, A. Rundquist, and J. Vučković, Phys. Rev. Lett. 108, 183601 (2012).
[42] T. Shi and S. Fan, Phys. Rev. A 87, 063818 (2013).

[43] E. Sánchez-Burillo, L. Martín-Moreno, J. García-Ripoll, and D. Zueco, arXiv:1602.05603.

[44] D. Horoshko, G. Patera, A. Gatti, and M. Kolobov, Eur. Phys. J. D 66, 239 (2012).

[45] C. Eichler, D. Bozyigit, C. Lang, M. Baur, L. Steffen, J. M. Fink, S. Filipp, and A. Wallraff, Phys. Rev. Lett. 107, 113601 (2011).

[46] G. W. Gardiner and P. Zoller, Quantum Noise, 2nd ed. (Springer-Verlag, Berlin, 2000).

[47] H. J. Carmichael, Statistical Methods in Quantum Optics 1, 2nd ed. (Springer, Berlin, 2002).

[48] T. Caneva, M. T. Manzoni, T. Shi, J. S. Douglas, J. I. Cirac, and D.E. Chang, New J. Phys. 17, 113001 (2015).

[49] S. Xu and S. Fan, Phys. Rev. A 91, 043845 (2015).

[50] Z.-L. Xiang, S. Ashhab, J. Q. You, and F. Nori, Rev. Mod. Phys. 85, 623 (2013).

[51] M. Devoret and R. Schoelkopf, Science 339, 1169 (2013).

[52] R. Schoelkopf and S. Girvin, Nature (London) 451, 664 (2008).

[53] J. Koch, T. M. Yu, J. Gambetta, A. A. Houck, D. I. Schuster, J. Majer, A. Blais, M. H. Devoret, S. M. Girvin, and R. J. Schoelkopf, Phys. Rev. A 76, 042319 (2007).

[54] J. A. Schreier, A. A. Houck, J. Koch, D. I. Schuster, B. R. Johnson, J. M. Chow, J. M. Gambetta, J. Majer, L. Frunzio, M. H. Devoret, S. M. Girvin, and R. J. Schoelkopf, Phys. Rev. B 77, 180502 (2008).

[55] J. D. Thompson, T. G. Tiecke, N. P. de Leon, J. Feist, A. V. Akimov, M. Gullans, A. S. Zibrov, V. Vuletic, and M. D. Lukin, Science 340, 1202 (2013). 